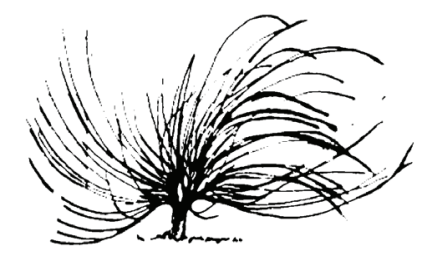

\title{
Actitud de estudiantes universitarias del área de nutrición hacia la ciencia
}

\author{
Pedro César Cantú-Martínez ${ }^{1}$ \\ Universidad Autónoma de Nuevo León \\ San Nicolás de los Garza, N.L., México \\ pedro.cantum@uanl.mx
}

\section{Resumen}

El presente artículo examina la actitud de estudiantes universitarias hacia la ciencia. Este estudio es de carácter descriptivo y comparativo, que se efectuó en la Universidad Autónoma de Nuevo León (Monterrey, N.L., México), de agosto a diciembre de 2014. Se realizó un muestreo no probabilístico en el que participaron 68 alumnas de 20 a 21 años del área de nutrición cuyo criterio de inclusión se contempló que hubieran cursado la materia de Bioética, que se ofrece en el séptimo semestre de la carrera profesional. Se utilizó el instrumento denominado Protocolo de Actitudes hacia la Ciencia (PAC). Se halló que las estudiantes participantes cuentan con un Índice de Actitud hacia la Ciencia de $73.21( \pm 5.91)$ en promedio que simboliza una buena actitud hacia la ciencia. Ostentándose que el $82.4 \%$ de las participantes tienen una buena actitud, y el $16.2 \%$ y el $1.5 \%$ de las

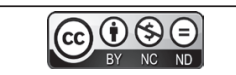

Recibido: 12 de diciembre de 2016-Aprobado: 3 de octubre de 2017

http://dx.doi.org/10.15359/rep.12-2.10

1 Doctor en Ciencias Biológicas. Profesor e investigador de la Universidad Autónoma de Nuevo León, adscrito a la Facultad de Ciencias Biológicas. Miembro del Cuerpo Académico 181 Ciencias Exactas y Desarrollo Humano. Miembro del Sistema Nacional de Investigadores Nivel 1 en el área de ciencias sociales. Su línea de investigación está relacionada con calidad de vida, desarrollo humano sustentable, bioética y educación. 
encuestadas poseen una actitud moderada y muy buena hacia la ciencia. Considerando esta evidencia se puede deducir que las jóvenes estudiantes manifiestan un buen interés y actitud a todo lo relacionado con la ciencia. Por otra, parte se puede determinar que esto es consecuencia del currículo escolar de orden formal como también del currículo oculto que aporta enseñanzas no explícitas a través de los distintos espacios y programas universitarios.

Palabras clave: actitud, ciencia, educación superior, estudiantes, nutrición.

\section{Abstract}

This article examines the attitudes of female university students towards science. This study is descriptive and comparative in nature, and it was conducted at the Universidad Autónoma de Nuevo León (Monterrey, N.L, México) from August to December of 2014. A convenience sample in which 68 students from 20 to 21 years old participated was conducted the area of nutrition whose inclusion criterion was they that had taken the subject of Bioethics, which is offered in the seventh semester of their major. The instrument called Protocol Attitudes towards Science (PAC) was used. It was found that the participating students have an index of attitude towards science of $73.21( \pm 5.91)$ on average symbolizing a good attitude towards science, showing that $82.4 \%$ of the participants have a good attitude, and $16.2 \%$ and $1.5 \%$ of respondents have a moderate to very good attitude towards science. Considering this evidence, it can be deduced that young students show a great interest and attitude towards everything related to science. On the other hand, it can be determined that this is a consequence of the formal school curriculum and hidden curriculum that also offers teachings that are not explicit through the university spaces and programs.

Keywords: attitude, science, higher education, students, nutrition. 


\section{Introducción}

En la actualidad la necesidad de evaluar las actitudes a los estudiantes universitarios en su trayectoria escolar es importante para la sociedad, esencialmente porque esto influye en su desempeño e incide en su profesión, donde el desafío es desarrollar en los niveles profesionales una condición indagadora e instruida en la ciencia como también en la tecnología (Plazas, Gómez y Castro, 2013). Por profesión acogeremos lo expresado por López-Suárez (2007, p. 13), que advierte que es "la facultad que adquiere una persona, usualmente como resultado de un largo proceso de formación escolarizada, para ejercer [...] una especialidad científica, tecnológica o humanística". Lo antes mencionado, conlleva a reconocer que dentro de este andar escolarizado por los educandos, que tiene como resultado la adquisición de una profesión, se suscitan en ellos una serie de comportamientos que involucran distintas valoraciones acerca de la información científica y tecnológica que se les proporciona durante su formación como profesionistas. A estas valoraciones se les denomina actitudes, que de acuerdo con Sabini, citado por Barra-Almagia (1998, p. 86), se constituyen en los elementos que vertebran en las personas "el pensamiento y la conducta". Esto es, la actitud "aunque no es directamente observable, precede a la conducta y guía nuestras elecciones y decisiones de acción” (Hogg y Vaughan, 2010, p. 139).

Las actitudes definidas por Briñol, Falces y Becerra (2007, p. $459)$ indican que son las "evaluaciones globales y relativamente estables que las personas hacen sobre otras personas, ideas o cosas que, técnicamente, reciben la denominación de objetos de actitud". Las actitudes son estructuralmente importantes en los individuos en el momento de adoptar nuevos conocimientos y comprensiones, porque son aspectos que se vinculan en forma permanente con el contexto social en que subsisten las personas, cuyas posturas manifiestas pueden ser tanto positivas, negativas como neutras o ambivalentes (García-Ruiz y Sánchez-Hernández, 2006; Hogg y Vaughan, 2010).

El interés por realizar estudios sobre actitudes hacia la ciencia se remonta hace años de manera relevante empleando "gran cantidad de tiempo y esfuerzos a la investigación [...] en sus más variados aspectos" como señalan Vázquez-Alonso y Manassero-Mas (1995, p. 337). Estas indagaciones realizadas hasta la actualidad han aportado valiosa información concerniente a las relaciones dialécticas entre las actitudes 
y lo conexo a la ciencia como son su naturaleza, contenidos, su enseñanza, las características de quién genera este conocimiento, entre otros aspectos; y se han llevado a cabo en distintas audiencias con diferentes niveles escolares que conciernen principalmente a examinaciones de carácter transversal y con variadas metodologías (Molina, Carriazo y Casas, 2013). Entre los trabajos más recientes podemos citar los publicados por García-Ruiz y Sánchez-Hernández (2006), Rodríguez, Jiménez y Caicedo-Maya (2007), Domínguez (2012), Hernández-Barbosa (2012), Rojas-Betancur, Méndez-Villamizar y Rodríguez-Prada (2012), Molina, Carriazo y Casas (2013), Plazas, Gómez y Castro (2013), Leal-Pérez, Hernández-Sampieri y Hernández-Bastida (2014), Pelcastre, Gómez y Zavala (2015).

Para favorecer al entendimiento de esta relación existente entre la ciencia y la actitud que poseen un grupo de estudiantes de educación superior, el presente estudio tiene como finalidad indagar sobre la actitud que tiene un grupo de estudiantes universitarias del área de nutrición con respecto a la ciencia, y determinar si existen diferencias significativas en el nivel de actitud hacia la ciencia tomando en consideración el empleo que harán de la información científica como actualización académica, investigación o ambas; como también conocer cuál es su inclinación profesional preponderante al recibir este conocimiento científico que residiría en la práctica clínica o bien desarrollar actividades como investigador.

\section{Marco conceptual}

Hoy, nuestra sociedad convive inmersa en un amplio contexto de posibilidades tecnocientíficas que se ostentan como columnas fundamentales del desarrollo económico y productivo, que inciden de forma general en la vida cotidiana (Cantú-Martínez, 2012a). En este escenario de modernidad, comprender el papel que la ciencia cumple es sumamente importante en esta globalidad contemporánea. Recordemos que gran parte del bienestar social que poseemos "se encuentra sustentado en la magnitud y privilegios que la ciencia y la técnica han conseguido para la humanidad" (Cantú-Martínez, 2012b, p. 35).

Manassero-Mas y Vázquez-Alonso (2001, p. 16) han advertido que "el movimiento didáctico ciencia-tecnología-sociedad (CTS) tiene como uno de sus objetivos el desarrollo de las actitudes relacionadas 
con la ciencia en el alumnado". Se ha enfatizado desde variadas posturas empíricas en materia de educación, pero particularmente en la educación superior, de la relevancia de atender no solo los elementos de carácter cognitivo que se ven implicados en los procesos de enseñanza-aprendizaje, sino también los de orden actitudinal, que están estrechamente vinculados a los elementos esgrimidos en la preparación profesional y al régimen de creencias como de costumbres que poseen los alumnos, de acuerdo con los entornos socioambientales de donde provienen. Recordemos que "se acepta que en la enseñanza de las ciencias intervienen variedad de conocimientos: el de ciencias, el del científico, el académico, el del profesor, el cotidiano, el del alumno" (Barros, 2008, p. 70). Esta conjunción de elementos se exterioriza en los estudiantes en una predisposición al aprendizaje y un reconocimiento consciente del conocimiento científico que circunda sus vidas, que más adelante se revela en inclinaciones actitudinales hacia la ciencia (Steinmann, Bosch y Aiassa, 2013).

La propensión de los trabajos de investigación sobre las posturas actitudinales hacia la ciencia ha conllevado distintas direcciones, entre las más recientes tenemos: la relacionada con la evaluación de la práctica docente (García-Ruiz y Sánchez-Hernández, 2006), la generación de instrumentos para evaluar las actitudes hacia la ciencia (Rodríguez, Jiménez y Caicedo-Maya, 2007), lo tocante sobre los significados y representaciones de la ciencia (Domínguez, 2012) y lo referente a las estimaciones actitudinales (Hernández-Barbosa, 2012; Rojas-Betancur, Méndez-Villamizar y Rodríguez-Prada, 2012; Molina, Carriazo y Casas, 2013; Plazas, Gómez y Castro, 2013; Leal-Pérez, Hernández-Sampieri y Hernández-Bastida, 2014; Pelcastre, Gómez y Zavala, 2015). Estos estudios mencionados en su conjunto demarcan que

además de aprender hechos, conceptos y principios de la ciencia, los estudiantes deben entender mejor cómo funciona ésta en el mundo actual, tanto en el ámbito interno (valores propios y naturaleza de la ciencia) como en el externo, en sus relaciones con la sociedad y la tecnología (valores contextuales y relaciones entre ciencia, tecnología y sociedad) (Vázquez-Alonso, Acevedo-Díaz y Manassero-Mas, 2001, p. 2). 
Para fortalecer la premisa anterior, podemos agregar de forma complementaria lo que Rodríguez-Gómez (2009, p. 67) asevera al señalar que los "procesos educativos deben abordar no sólo conocimientos y destrezas sino aquellos valores y actitudes que promuevan la integración del educando en un mundo en permanente cambio". Para esto es necesario apuntalar una actitud idónea en los estudiantes, para lo cual es provechoso y conveniente por parte de los educadores tener injerencia en su bagaje de conocimientos, en ahondar en las condiciones de sus preferencias y examinar las particularidades conductuales de estos (Hernández, Gómez, Maltes, Quintana et al., 2011).

Lo antes aludido es de suma importancia ya que los estudiantes por lo general suelen estar boyantes con sus propias ideas y quedarse en estas posturas particulares para estructurar una argumentación del contexto en lo que han sido ilustrados. Sin embargo, hacen hincapié Campanario, Cuerva, Moya y Otero (1998), que en muchas de las ocasiones estos posicionamientos personales suelen estar distanciados de la realidad expresada por sus mentores y de las proclamas estudiadas. Por lo tanto, se torna pertinente señalar que "la enseñanza de la ciencia se debe contextualizar acorde a las realidades de los alumnos, a sus entornos inmediatos, en los que ellos puedan intervenir creando y solucionando problemas de la vida cotidiana" (Hernández, Gómez, Maltes, Quintana et al., 2011, p. 72); al hacer acopio de los conocimientos recibidos, además de vincularlos a las múltiples experiencias y relaciones que sus educadores les han mostrado.

Es así que resulta muy conveniente y pertinente indagar las posturas actitudinales que manifiestan los alumnos para comprender el peso de las acciones académicas y extracurriculares asumidas en su formación, que tienen como finalidad mejorar su actuación y comprensión del ambiente tanto social como profesional en el que se desenvolverán.

\section{Metodología}

La presente investigación es de carácter descriptivo por tanto es un estudio en el que se han seleccionado variables que se cuantifican con la finalidad de describirlas, y es comparativa ya que nos permite fortalecer nuestra capacidad de descripción al proponernos contrastar las relaciones que se manifiestan en derredor de medir las Actitudes hacia la Ciencia que muestran mujeres universitarias estudiantes de la 
carrera de Licenciatura en Nutrición, en la Universidad Autónoma de Nuevo León (Monterrey, N.L., México). El levantamiento de los datos se realizó de agosto a diciembre de 2014. La selección de las participantes fue de carácter no probabilístico tomando una muestra intencional de 68 estudiantes universitarias. Como criterio de inclusión se contempló que hubieran cursado la materia de Bioética, que se ofrece en séptimo semestre de la carrera profesional, en cuyo contenido se escudriña y reflexiona sobre los aspectos que atañen a la investigación científica y en salud, como también el considerar la investigación científica como un documento éticocientífico. Las edades de las participantes fluctuaron entre 20 y 21 años.

Se aplicó el instrumento denominado Protocolo de Actitudes hacia la Ciencia (PAC) adecuado por Rodríguez, Jiménez y Caicedo-Maya, en el año 2007, a partir del trabajo inicial realizado por Vázquez-Alonso y Manassero-Mas en el año de 1995 y 1997. Este consta de 50 cuestionamientos que contemplan cuatro dimensiones con respecto a la ciencia: que observan las dimensiones "Enseñanza de la Ciencia", "Imagen Social de la Ciencia", "Dimensión Social e Incidencia de la Ciencia" y "Característica de la Ciencia". Asimismo, involucra cinco subdimensiones que son: "Ciencia Escolar", "Resultados de la Ciencia", "Características de la Ciencia" "Construcción Colectiva de la Ciencia" y la "Naturaleza de la Ciencia"; las cuales dan cuenta de las actitudes hacia los componentes y productos del aprendizaje de la ciencia y tecnología, con las interacciones con la sociedad en lo referente a la particularidad colectiva, valores y procedimientos científicos.

Este instrumento consta de una escala de Likert que involucra cinco respuestas posibles que van de totalmente en desacuerdo a totalmente en de acuerdo, en una escala de 1 a 5 , para calificar las respuestas. El cuestionario abarca cuestionamientos que están enunciados de forma positiva y otros de manera negativa, las escalas de las preguntas negativas se han determinado numéricamente en sentido inverso, de modo que las puntuaciones elaboradas indiquen que los valores mayores representan una mejor condición actitudinal hacia la ciencia. Se codificaron los datos posteriormente para su tratamiento estadístico en el programa SPSS; las variables cualitativas se examinaron mediante frecuencias y las de carácter cuantitativo con medidas de tendencia central y prueba de $t$ Student para los valores medios. Luego se empleó un baremo para determinar el grado de actitud que mostraron las estudiantes, el cual 
consta de la siguiente escala: 20 a 36 "muy baja"; 36 a 52 "baja"; 52 a 68 "moderada"; 68 a 84 "buena"; 84 a 100 "muy buena". La confiabilidad del instrumento administrado en esta investigación fue de $81.6 \%$ de acuerdo con el Alfa de Cronbach. La presente indagación se apegó a lo pertinente en la Ley General de Salud en Materia de Investigación para la Salud (EUM, 1986) y se contó con la aprobación de las participantes para intervenir en la presente investigación catalogada como sin riesgo.

\section{Resultados}

\subsection{Actitud hacia la Ciencia}

La Actitud hacia la Ciencia, que se manifiesta por la muestra de 68 estudiantes universitarias del área de nutrición, presenta un valor promedio de 73.21 con una variación de los datos con respecto al valor medio encontrado de 5.91, que cotejándolo con los intervalos del baremo que se desarrolló, la puntuación se encuentra en una catalogación de actitud "buena" (ver Tabla 1). Lo anterior representa una actitud favorable hacia la ciencia, que también se puede constatar de manera agrupada, ya que es ostensible observar que la mayoría de las encuestadas se encuentran en una catalogación de actitud "buena" que representa un $82.4 \%$, continuando seguidamente en menor porcentaje la actitud "moderada" y "muy buena", con valores que representan el $16.2 \%$ y el $1.5 \%$, respectivamente.

En el marco del análisis de los cuestionamientos, las actitudes más positivas están representadas en la Dimensión Social de la Ciencia, donde cuatro cuestionamientos recibieron un puntaje promedio superior a 4, con lo cual se puede corroborar que las estudiantes consideran que la ciencia aporta un mundo mejor, que esta sí contribuye de manera sustanciosa al adelanto en la sociedad para ser más efectivo en tiempo y esfuerzo en los procesos en que interviene el ser humano, además que la ciencia ha evitado muchas enfermedades y ha permitido tener grandes avances y progresos sociales. En este mismo sentido continúan las Dimensiones de Enseñanza de la Ciencia y Características de la Ciencia, con tres cuestionamientos cada una que recibieron un puntaje promedio superior a 4. Que en primera instancia permite fortalecer el hecho que las estudiantes consideran pertinente el que existan asignaturas de ciencia, con la finalidad de vigorizar la trayectoria académica de los 
estudiantes y, por lo tanto, la ciencia es conveniente para la sociedad; y en segundo término, que la ciencia es el resultado de la indagación y escudriñamiento por los científicos, donde las nuevas opiniones son importantes para su desempeño y progreso. Finalmente, se encuentra la Dimensión Imagen Social de la Ciencia, en la cual dos cuestionamientos concluyeron con valores numéricos mayores a 4 en promedio, que permiten vislumbrar que las participantes observan que la ciencia les es de sumo interés a las personas y su contribución es muy valiosa.

\subsection{Dimensiones y Subdimensiones}

\subsubsection{Dimensión Enseñanza de la Ciencia}

En lo referente a la actitud hacia los elementos de Enseñanza y Aprendizaje de la Ciencia que involucra elementos escolares y productos esperados del aprendizaje, por las participantes en el estudio, reveló que el 82.4\% ( $f=56)$ muestra una actitud "buena", el 16.2\% ( $f=11)$ "moderada" y solo el 1.5\% ( $\mathrm{f}=1)$ "muy buena". Además se observaron calificaciones que fluctuaron entre 50.77 y 87.69 , con un promedio de 72.12 y una variación de los datos de 8.38 , cifra que permite aseverar que la población investigada cuenta con una actitud promedio hacia la Enseñanza de la Ciencia de "buena".

\subsubsection{Subdimensión Resultados de la Ciencia}

En cuanto a la actitud hacia los Resultados de la Ciencia, que se constituyen en productos que demuestran la transferencia de la ciencia y tecnología asimilada en aspectos de la vida diaria, hallamos que el $66.2 \%(f=45)$ exhibe una actitud "buena", el 17.6\% ( $f=12)$ "moderada", el 10.3\% ( $\mathrm{f}=7)$ "muy buena" y el 5.9\% $(\mathrm{f}=4)$ una condición "baja". Conjuntamente se averiguó que las evaluaciones en el comportamiento actitudinal hacia los Resultados de la Ciencia oscilaron entre 48.57 y 88.57 , con un promedio de 73.27 y una variación de 9.90 , resultado que evidencia una actitud promedio considerada como "buena" para dicha población.

\subsubsection{Subdimensión de Ciencia Escolar}

En lo que respecta hacia la Ciencia Escolar, que advierte de las actitudes que demuestran las estudiantes en su contexto institucional, se denota que el $45.6 \%(f=31)$ exhibe una actitud "moderada", el 39.7\% 
$(f=27)$ "buena", el 10.3\% ( $f=7)$ "muy buena" y el 4.4\% ( $f=3)$ "baja". Asimismo, se apreció que los valores de actitud hacia la enseñanza escolar de la ciencia variaron entre 46.67 y 93.33 , con un promedio de 70.78 y una variación de 11.47 , lo cual evidencia que la población cuenta con una actitud promedio estimada como "buena".

\subsubsection{Dimensión Imagen Social de la Ciencia}

En cuanto a la actitud hacia la Imagen Social de la Ciencia que indica la interacción de la sociedad con asuntos científicos, el 61.8\% (f $=42)$ cuenta con una actitud "buena", el 29.4\% $(\mathrm{f}=20)$ "moderada", el $7.4 \%(\mathrm{f}=5)$ "muy buena" y el 1.5\% ( $\mathrm{f}=1)$ "baja". También se distinguieron calificaciones actitudinales que fluctuaron entre 51.11 y 88.89 , con un promedio de 72.22 y una variación de los datos de 8.22 , cifra de la que se deduce que la población investigada cuenta con una actitud "buena" en promedio.

\subsubsection{Dimensión Social e Incidencia de la Ciencia}

En lo relativo a la actitud hacia lo Social e Incidencia de la Ciencia, que expresa la importancia de la transferencia de la ciencia y tecnología, el 69.1\% ( $\mathrm{f}=47$ ) la manifiesta "buena", el 23.5\% ( $\mathrm{f}=$ 16) "moderada" y el 7.4\% ( $\mathrm{f}=5)$ "muy buena". A la par se examinó que las evaluaciones de actitud oscilaron entre 58.75 y 88.75 , con un promedio de 73.67 y una variación de 7.06 , lo cual, respecto de esta dimensión, evidencia que la población cuenta con una "buena" actitud en promedio.

\subsubsection{Dimensión Característica de la Ciencia}

En cuanto a la actitud hacia la Característica de la Ciencia, que representa los métodos, ideología y forma de construcción de la ciencia, reveló en las alumnas que el 76.5\% ( $\mathrm{f}=52)$ tuvo una actitud "buena", el $13.2 \%(\mathrm{f}=9)$ "moderada", el $8.8 \%(\mathrm{f}=6)$ "muy buena" y, finalmente, el 1.5\% ( $\mathrm{f}=1)$ "baja". Se observó que los valores de actitud oscilaron entre 50 y 90 , con un promedio de 74.53 y una variación de 7.68 , cifra de la que se concluye que la población cuenta con una "buena" actitud hacia las características de la ciencia. 


\subsubsection{Subdimensión Naturaleza de la Ciencia}

En lo concerniente a la actitud hacia la Naturaleza de la Ciencia, que simboliza los métodos y productos propios del conocimiento científico, se comprobó en las educandas que el $66.2 \%(f=45)$ cuenta con una "buena" actitud, el 25\% ( $\mathrm{f}=17$ ) "moderada", continuando con el 4.4\% ( $f=3$ ) para una postura "muy buena" y "baja". Se observaron calificaciones sobre la actitud que fluctuaron entre 43.33 y 90 , con un promedio de 70.98 y una variación de los datos de 8.90 , cifra de la que es correcto afirmar que la población investigada cuenta con una "buena" actitud en promedio.

\subsubsection{Subdimensión de Características de la Ciencia}

En lo comprendido a la actitud hacia las Características de la Ciencia, que demuestra la ideología y cualidades personales de los científicos, reveló en las estudiantes que el $44.1 \%(\mathrm{f}=30)$ tiene una postura "buena", el 42.6\% ( $f=29)$ "muy buena", el 11.8\% $(f=8)$ "moderada" y el $1.5 \%$ ( $\mathrm{f}=1)$ "baja". Se constató que las valoraciones actitudinales variaron entre 45 y 100 , con un promedio de 70.82 y una variación de 10.65 , resultado que demuestra una actitud "buena" en promedio.

\subsubsection{Subdimensión Construcción Colectiva de la Ciencia}

En lo tocante a la actitud hacia la Construcción Colectiva de la Ciencia, que atañe a la construcción del conocimiento científico, las estudiantes encuestadas mostraron una actitud "buena" en un $52.9 \%$ $(\mathrm{f}=36)$, "muy buena" en un $25 \%(\mathrm{f}=17)$, "moderada" en un $14.7 \%$ $(\mathrm{f}=10)$, "baja" en un $5.9 \%(\mathrm{f}=4)$ y en un $1.5 \%(\mathrm{f}=1)$ "muy baja". Se verificó que las valoraciones actitudinales permanecieron entre $30 \mathrm{y}$ 100 , con un promedio de 76.61 y una variación de 14.41, resultado que demuestra una actitud "buena" en promedio.

\subsection{Correlación entre el Î́ndice de Actitud hacia la Ciencia y las di- mensiones y subdimensiones evaluadas}

En lo que respecta al Índice de Actitud hacia la Ciencia y su asociación con las dimensiones y subdimensiones evaluadas de cada una de las estudiantes universitarias, se evidenciaron correlaciones estadísticamente significativas con ellas (ver Tabla 1). 
Tabla 1. Correlación del Î́ndice de Actitud hacia la Ciencia con las Dimensiones y Subdimensiones de estudiantes universitarias del área de nutrición

\begin{tabular}{|l|c|c|}
\hline \multicolumn{1}{|c|}{ Índice de Actitud hacia la Ciencia } & $\begin{array}{c}\text { Correlación } \\
\text { de Pearson }\end{array}$ & Significancia \\
\hline Dimensión Enseñanza de la Ciencia & 0.795 & $<0.01$ \\
\hline Subdimensión Resultados de la Ciencia & 0.741 & $<0.01$ \\
\hline Subdimensión de Ciencia Escolar & 0.531 & $<0.01$ \\
\hline Dimensión Imagen Social de la Ciencia & 0.750 & $<0.01$ \\
\hline Dimensión Social e Incidencia de la Ciencia & 0.777 & $<0.01$ \\
\hline Dimensión Característica de la Ciencia & 0.715 & $<0.01$ \\
\hline Subdimensión Naturaleza de la Ciencia & 0.518 & $<0.01$ \\
\hline Subdimensión de Características de la Ciencia & 0.543 & $<0.01$ \\
\hline Subdimensión Construcción Colectiva de la Ciencia & 0.525 & $<0.01$ \\
\hline
\end{tabular}

Fuente: Elaboración propia del autor.

\section{4 Índice de Actitud hacia la Ciencia y su contraste con la inclina- ción profesional, empleo de la información científica}

En relación con el uso de la información científica, el 19.1\% (f= 13) la utilizarán para investigación, mientras que aquellas estudiantes que reportan práctica clínica, el $54.4 \%$ ( $\mathrm{f}=37)$ la emplearán solo para la actualización de conocimientos como lo declaran, y por otra parte el $26.4 \%(f=18)$ argumentan que les servirá preferentemente para la actualización de su práctica clínica y en algunas ocasiones para realizar investigación. Con respecto a los valores promedio del Índice de Actitud hacia la Ciencia, estos se significaron en valores de $73.10( \pm 5.28)$, $73.01( \pm 5.96)$ y $73.71( \pm 6.53)$, que conciernen a las afirmaciones de investigación, actualización de conocimientos de la práctica clínica, y actualización de su práctica clínica, como de vez en cuando realizar investigación, respectivamente. Que permitió catalogarlas con una actitud "buena", no encontrando diferencia significativa entre estos valores a un $95 \%$ de confiabilidad. Similar pauta fue hallada en las distintas dimensiones y subdimensiones consideradas.

Finalmente, con respecto a su inclinación profesional, el 80.8\% $(\mathrm{f}=55)$ estudiantes expresan que se dedicarán a la práctica clínica y solo el 19.1\% ( $\mathrm{f}=13)$ pretenden continuar en actividades relacionadas 
con la investigación. No obstante, esta diferencia porcentual, la actitud exteriorizada por las encuestadas indica un Índice de Actitud hacia la Ciencia que se establece en valores promedio de $73.24( \pm 6.10)$ y 73.10 $( \pm 5.28)$, que respectivamente corresponden a una catalogación actitudinal "buena" a quienes realizarán práctica clínica e investigación. No encontrándose diferencia significativa con un $95 \%$ de confiabilidad entre estos valores medios, producto de su preferencia profesional. Mostrándose este mismo comportamiento para todas las dimensiones y subdimensiones.

\section{Discusión de resultados}

Se puede aseverar que las jóvenes universitarias que accedieron a participar en el estudio cuentan con una "buena" actitud, que configura una postura favorable y positiva hacia la ciencia, que de acuerdo con Plazas, Gómez y Castro (2013), estas preferencias actitudinales se ven grandemente favorecidas si en el contexto en que discurren su trayectoria académica las alumnas cuentan con componentes curriculares que refieran al método científico y espacios para desempeñar y acercarse a la actividad científica de manera formal. Recordemos que la ciencia es un proceso donde las acciones y conductas dependen del contexto social (González-Guitián y Molina-Piñeiro, 2008; Cantú-Martínez, 2012a; Leal-Pérez, Hernández-Sampieri y Hernández-Bastida, 2014). Y en el caso de la universidad, de acuerdo con Rojas-Betancur, Méndez-Villamizar y Rodríguez-Prada (2012, p. 217), esta tarea es de carácter transcendental ya que ha de "permear las estructuras curriculares y la cotidianidad educativa hacia la construcción de una cultura de la investigación [y científica]”.

Estos aspectos antes citados se ven presentes en la realidad de las alumnas participantes en el estudio, debido que la Universidad Autónoma de Nuevo León (UANL), en su marco axiológico y en particular en su misión, denota la pertinencia como institución de educación superior de crear conocimiento social, científico y humanista, aunado a la formación de recursos humanos, a los cuales los hace participes de este conocimiento (UANL, 2011). En su escenario institucional, cuenta con variados espacios para la inserción de las estudiantes de pregrado en actividades científicas, ya que tiene 38 Centros e Institutos de Investigación y Desarrollo de la Ciencia, Cultura y el Arte. Además 
posee el Programa de Verano de Investigación Científica y Tecnológica-UANL, en el cual el alumno de pregrado participa por cuatro o cinco semanas, en un horario completo en proyectos de investigación para tener contacto personal con investigadores, con la finalidad de definir su vocación científica, por otra parte, se encuentra el Programa de Apoyo a la Investigación Científica y Tecnológica-UANL, espacio donde los estudiantes pueden integrarse a proyectos de investigación mediante el involucramiento con un investigador, ya que entre las premisas de este programa se menciona que deben participar estudiantes de pregrado (UANL, 2016). Conjuntamente a estos programas anuales los estudiantes también logran intervenir como becarios en apoyo a los investigadores de manera permanente en sus respectivas dependencias donde cursan su carrera universitaria.

Es de destacar la interacción de estos programas institucionales en los procesos de enseñanza aprendizaje de la ciencia, los cuales inciden en el marco áulico y, por ende, en la actitud que muestran los estudiantes hacia la ciencia. En este orden de ideas, Hernández-Barbosa (2012, p. 99) indica que "lo anterior también puede estar relacionado con otros aspectos que se inscriben en el ambiente escolar y particularmente a uno de sus agentes: el profesor". A este respecto Ríos-Cabrera y Ruedas-Marrero (2008, p. 193) señalan que el profesor "es quien diseñará estrategias de enseñanza dirigidas a sus estudiantes, si están bien orientadas, ayudarán a que éstos comprendan las interacciones de la ciencia y la tecnología, fortaleciendo y desarrollando valores".

En este sentido, consideramos que han incidido estos factores en los puntajes promedio, que fueron muy similares entre el Índice de Actitud hacia la Ciencia como también en cada una de las dimensiones y subdimensiones que se examinaron en el instrumento, dando un resultado actitudinal que permite observar en las alumnas la integración del conocimiento científico y tecnológico que irrumpe en sus vidas. Domínguez (2012) y Pelcastre, Gómez y Zavala (2015) estipulan en sus investigaciones que en México se cuenta con una pobre cultura científica; debemos juzgar por cultura científica, de acuerdo con Cantú-Martínez (2012a, p. 31), como el acto en el cual se anhela que

CITAlos destinatarios últimos, las personas, no sólo obtendrán información, sino además una preparación para obtener habilidades y aptitudes que les permitan situar y reconocer el conocimiento científico 
en sus vidas, y con ello, les dejará trascender en diferentes recintos estructurados en nuestra sociedad.

Considerando este pronunciamiento, las jóvenes estudiantes en este estudio han manifestado un buen interés y actitud hacia lo relativo con la ciencia, que permitió identificar como señala Solbes y Vilches (1989, p. 19), "que el alumno tenga una visión real de la ciencia y su relación con la técnica y la sociedad". Es así que la ciencia transmitida mediante el proceso de cultura científica a las alumnas se ha de constituir en un "recurso para el pleno desarrollo y ejercicio de la ciudadanía del estudiante" (Rojas-Betancur, Méndez-Villamizar y Rodríguez-Prada, 2012, p. 217), en un marco de referencia que encamine a la movilización conveniente y real del empoderamiento de las competencias científicas y tecnológicas (Levy, 2004).

\section{Conclusiones}

La presente investigación nos ha mostrado evidencias que están acordes con los resultados de otros estudios. Es así, que el posicionamiento de las estudiantes en una actitud positiva hacia la ciencia es resultado del entorno formativo, el cual se encuentra contextualizado en la vida social, y cuyos conocimientos como acciones están centrados en la búsqueda de resolución de aspectos de interés social. Es importante subrayar, que si vinculamos este resultado con el marco axiológico y programas con los que cuenta la UANL, podemos percibir que la buena actitud mostrada por las alumnas es también consecuencia del currículo escolar de carácter formal que se encuentra plasmado en el plan de estudios, como también del currículo oculto que entrega enseñanzas no explícitas a través de los espacios universitarios que se generan para el involucramiento de las estudiantes con la ciencia. Que las preparan tanto para su vida personal como profesional, con una postura realista frente al ejercicio de su actividad profesional con apropiamiento de las capacidades y habilidades para desenvolverse adecuadamente frente a los avances científicos y tecnológicos, y con un alto compromiso social. 


\section{Referencias}

Barra-Almagia, E. (1998). Psicología Social. Chile: Universidad de Concepción.

Barros, J. F. (2008). Enseñanza de las ciencias desde una mirada de la didáctica de la escuela francesa. Revista EIA, 10, 55-71.

Briñol, P., Falces, C. y Becerra, A. (2007). Actitudes En J. F. Morales, M.C. Moya, E. Gaviria \& I. Cuadrado (Coords.), Psicología Social (pp. 457-490). España: McGraw-Hill.

Campanario, L., Cuerva, M., Moya L. y Otero, G. (1998). La metacognición y el aprendizaje de las ciencias. En E. Banet y A. de Pro (Coords.), Investigación e innovación en la enseñanza de las ciencias. Vol. I (pp. 36-44). Murcia: DM.

Cantú-Martínez, P. C. (2012a). Discurrir y apropiamiento de la ciencia por la sociedad. En P.C. Cantú-Martínez (Ed.), Sustentabilidad Cientifica. Introversión sobre la Ciencia, Conciencia y Racionalidad Social (pp. 25-32). México: Clave Editorial y Universidad Autónoma de Nuevo León.

Cantú-Martínez, P.C. (2012b). Ética en la sociedad tecnocientifica En P.C. Cantú-Martínez (Ed.), Sustentabilidad Cientifica. Introversión sobre la Ciencia, Conciencia y Racionalidad Social (pp. 3544). México. Clave Editorial-Universidad Autónoma de Nuevo León

Domínguez, S. (2012). Significados de la ciencia en estudiantes universitarios: aproximaciones a las representaciones sociales de la ciencia, del cientifico y de la actividad cientifica. Guadalajara: Universidad de Guadalajara.

Estados Unidos Mexicanos (EUM) (1986). Reglamento de la Ley General de Salud en Materia de Investigación en Salud. México: Diario Oficial de la Federación.

García-Ruiz, M. y Sánchez-Hernández, B. (2006). Las actitudes relacionadas con las ciencias naturales y sus repercusiones en la práctica docente de profesores de primaria. Perfiles Educativos, 28(114), 61-89.

González-Guitián, M.V. y Molina-Piñeiro, M. (2008). La evaluación de la ciencia y la tecnología: revisión de sus indicadores. Acimed, 8(6). Recuperado de http://www.bvs.sld.cu/revistas/aci/ vol18_6_08/aci031208.pdf 
Hernández-Barbosa, R. (2012). Actitudes hacia la ciencia en estudiantes de grado undécimo de algunos colegios públicos y privados de Bogotá. Revista de la Facultad de Psicología Universidad Cooperativa de Colombia. 8(14), 93-103.

Hernández, V., Gómez, E., Maltes, L., Quintana, M., Muñoz, F., Toledo, H., Riquelme, V., Henríquez, B., Zelada, B. y Pérez, E. (2011). La actitud hacia la enseñanza y aprendizaje de la ciencia en alumnos de Enseñanza Básica y Media de la Provincia de Llanquihue, Región de Los Lagos-Chile. Estudios Pedagógicos, 37(1), 71-83.

Hogg, M. A. y Vaughan, G. M. (2010). Psicología social. España: Editorial Médica Panamericana.

Leal-Pérez, B. E., Hernández-Sampieri, R. y Hernández-Bastida, A. (2014). Actitudes asociadas a la ciencia en la educación básica en México. Estudios en Ciencias Sociales y Administrativas de la Universidad de Celaya. 4(1), 40-57.

Levy, P. (2004). Inteligencia colectiva: por una inteligencia del ciberespacio. Washington: BIREME-OPS-OMS.

López-Suárez, A. (2007). Certificación y control de la práctica profesional: Una delimitación conceptual y operativa En M. Córdova Osnaya y J. C. Rosales Pérez (Coords.), Psicología Social. Perspectivas y aportaciones hacia un mundo posible (pp. 12-21). México: AMAPSI Editorial.

Manassero-Mas, M.A. \& Vázquez-Alonso, A. (2001). Instrumentos y métodos para la evaluación de las actitudes relacionadas con la ciencia, la tecnología y la sociedad. Enseñanza de las Ciencias. 20(1), 15-27.

Molina, M., Carriazo, J. y Casas, J. (2013). Estudio transversal de las actitudes hacia la ciencia en estudiantes de grados quinto a undécimo. Adaptación y aplicación de un instrumento para valorar actitudes. TED: Tecné, Episteme y Didaxis, 33, 103-122.

Pelcastre, L., Gómez, A. R. y Zavala, G. (2015). Actitudes hacia la ciencia de estudiantes de educación preuniversitaria del centro de México. Revista Eureka sobre Enseñanza y Divulgación de las Ciencias. 12(3), 475-490.

Plazas, M., Gómez, M. y Castro, C. A. (2013). Actitud en estudiantes de Ciencias de la Salud hacia el conocimiento científico. Rev. Cienc. Salud., 11(1), 83-91. 
Ríos-Cabrera, M. M. y Ruedas-Marrero, M. J (2008). Enseñanza de la ciencia para un futuro sostenible. Multiciencias. 8(Número Extraordinario), 192-197.

Rodríguez-Gómez, J. M. (2009). Cambios metodológicos relacionados con el aprendizaje de las ciencias. Revista Educación 33(1), 61-73.

Rodríguez, W., Jiménez, R. y Caicedo-Maya C.A. (2007). Protocolo de actitudes relacionadas con la ciencia: Adaptación para Colombia. Psicología: Avances en la Disciplina, 1(2), 85-100.

Rojas-Betancur, H.M., Méndez-Villamizar, R. y Rodríguez-Prada, A. (2012). Índice de actitud hacia la investigación en estudiantes del nivel de pregrado. Entramado, 8(2), 216-229.

Solbes, J. y Vilches, A. (1989). Interacciones ciencia/técnica/sociedad: Un instrumento de cambio actitudinal. Enseñanza de las Ciencias, 7(1), 14-20.

Steinmann, A., Bosch, B. y Aiassa, D. (2013). Motivación y expectativas de los estudiantes por aprender ciencias en la universidad. Revista Mexicana de Investigación Educativa, 18(57), 585-598.

Universidad Autónoma de Nuevo León (UANL). (2011). Visión 2020UANL. San Nicolás de los Garza: UANL.

Universidad Autónoma de Nuevo León (UANL). (2016). Investigación-Convocatorias y Apoyos. Recuperado de http://www.uanl. $\mathrm{mx} / \mathrm{universidad/investigacion/apoyos/apoyos.html}$

Vázquez-Alonso, A. y Manassero-Mas, M. A. (1995). Actitudes relacionadas con la ciencia: Una revisión conceptual. Enseñanza de las Ciencias, 13(3), 337-346.

Vázquez-Alonso, A. y Manassero-Mas, M. A. (1997). Una evaluación de las actitudes relacionadas con la ciencia. Enseñanza de las Ciencias, 15(2), 199-213.

Vázquez-Alonso, A., Acevedo-Díaz, J. A. y Manassero-Mas, M. A. (2001). Enseñando ciencia: consenso y disenso en la educación y evaluación de las actitudes relacionadas con la ciencia. Recuperado de http://www.campus-oei.org/salactsi/vazquez.htm 